\title{
A Case Report: Cutaneous Metastasis of Advanced Rectal Cancer with BRAF Mutation
}

This article was published in the following Dove Press journal:

OncoTargets and Therapy

\author{
Shishi Zhou \\ Wanfen Tang \\ Qinghua Wang \\ Xia Zhang \\ Xiayun Jin \\ Xifeng $\mathrm{Xu}$ \\ Jianfei Fu (D)
}

Department of Medical Oncology, Jinhua Hospital, Zhejiang University School of Medicine, Jinhua, 321000, Zhejiang

Province, People's Republic of China
Correspondence: Jianfei Fu

Department of Medical Oncology, Jinhua Hospital, Zhejiang University School of

Medicine, Jinhua, 321000, Zhejiang

Province, People's Republic of China

Email I I218276@zju.edu.cn

\begin{abstract}
Cutaneous metastasis of rectal cancer is rare and typically indicates widespread disease and poor prognosis. We report an exceedingly rare case of BRAF-mutated MSS rectal cancer with metastasis to the skin. A 53-year-old woman presented with stage IV unresectable adenocarcinoma of the rectum and received chemotherapy and molecularly targeted agents. Six months later she developed a focal skin nodule in the left groin. During treatment with four cycles of FOLFIRI plus bevacizumab, the skin nodules gradually increased in size, involving the skin of the left thigh. A portion of the rash was bleeding and painful. The biopsy specimen was consistent with a mucinous adenocarcinoma of rectal origin and expressed reduced CDX-2. Palliative treatment with FOLFIRI plus cetuximab and vemurafenib was initiated. The cutaneous nodules decreased in size but were not stable. The patient had severe electrolyte disturbances and depression and opted for palliative care.
\end{abstract}

Keywords: rectal cancer, cutaneous metastasis, BRAF-mutated, MSS, CDX-2

\section{Introduction}

Colorectal cancer (CRC) is the most common cancer and is the second most common cause of cancer-related deaths in 2015, after lung cancer. Approximately $30 \%$ of CRC patients present with metastatic disease, while the remaining patients are at risk of developing distant relapses. ${ }^{1}$ Metastases to the skin from an internal malignancy are uncommon. The most common sources of cutaneous metastases are lung and breast cancers, as well as melanoma. ${ }^{2}$ Skin metastases of adenocarcinoma of the rectum are even more rare, occurring in $<4 \%$ of patients. The median survival of patients after the appearance of cutaneous metastatic lesions is $18-20$ months. ${ }^{3}$ The gross appearance of skin metastases is usually solid and small $(<5 \mathrm{~cm})$ painless nodules or papules. ${ }^{4}$ Skin metastases can mimic cysts, lipomas, granulomas, and neurofibromas. Therefore, biopsy of suspicious lesions is essential. ${ }^{3}$

Activating mutations of BRAF are found in 5\%-9\%of colorectal carcinomas. Patients with BRAF-mutated MSS colorectal carcinomas are most often stage IV. ${ }^{5}$ Cytokeratin 20 (CK20) and caudal-type homeobox 2 (CDX-2) are two of the most sensitive and specific markers of intestinal differentiation and are extremely useful immunohistochemical markers in correctly identifying adenocarcinomas of colorectal origin in the metastatic setting. BRAF-mutated MSS colorectal carcinoma often displays reduced $\mathrm{CDX}-2^{6}$.

We report an uncommon case of skin metastases during the treatment of advanced rectal cancer and elucidate the internal relationship between cutaneous metastasis and reduced CDX-2 expression in rectal cancer. 


\section{Case Report}

A 53-year-old woman who presented with mucosanguineous feces and tenesmus was admitted to Jinhua Central Hospital in January 2019. A cauliflower-like mass occupied one-third of the rectal circumference $6 \mathrm{~cm}$ from the anus. The lumen of the intestine was visualized $8-15 \mathrm{~cm}$ with an endoscope. The pathologic diagnosis of an endoscopic biopsy was rectal adenocarcinoma (Figure 1A). Viewed under electron microscopy, the adenocarcinoma rectal tissue consisted of pleomorphic epithelial cell and a large number of lymphocytic, the neoplastic glands have crowded nuclei with pleomorphism, the cytoplasm was abundant. And immunohistochemical analysis showed CDX2(-) (Figure 1B). A contrastenhanced MRI showed that there were multiple lesions metastatic to the liver. A BRAF-V600E mutation and RAS were detected in the primary lesion. The status of microsatellites was stable. The clinical AJCC TNM classification staging was T3N2M1. The patient received five cycles of FOLFOX plus cetuximab from January to April 2019 and achieved a stable response (SD). In September 2019, the skin of the hypogastrium and perineum was red and swollen with a rubbery appearance, a rash-like swelling on the surface,

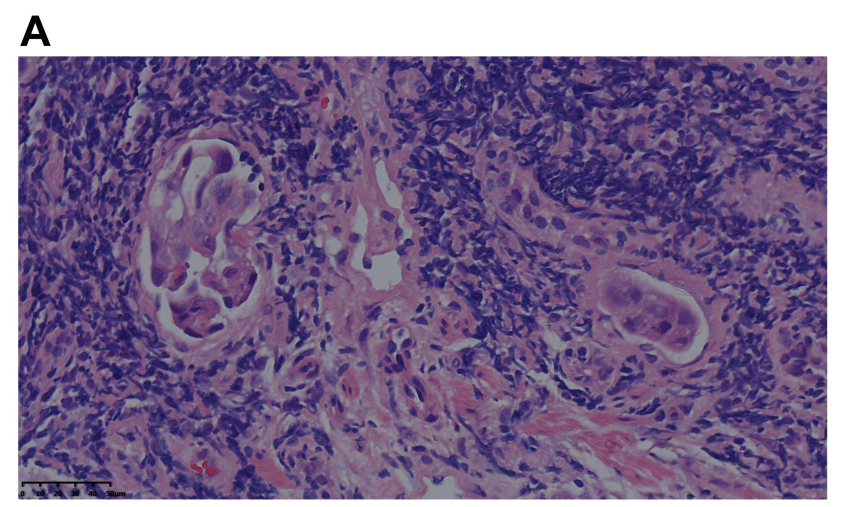

B

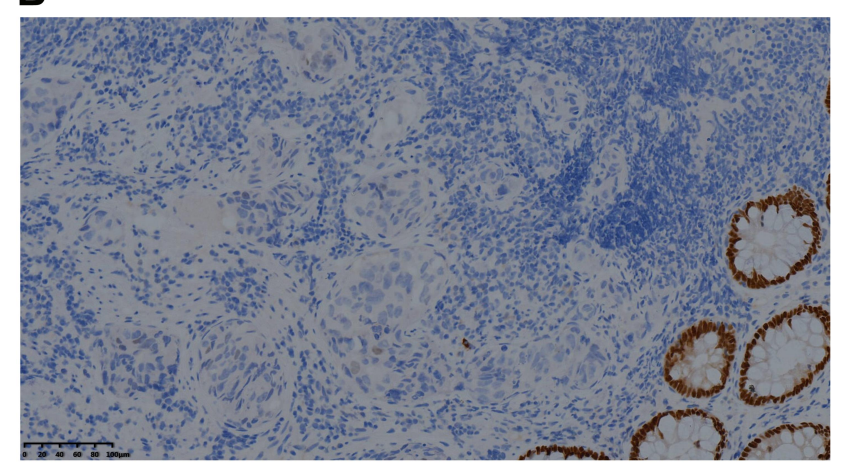

Figure I The pathologic diagnosis of an endoscopic biopsy of rectal.

Notes: Neoplasm composed of pleomorphic epithelial cells arranged in glandular pattern (hematoxylin and eosin staining, $\times 40$ ) (A); Immunohistochemical exam positive for CDX2 (B). and local fusion (Figure 2A). Progressive disease (PD) was demonstrated based on positron emission tomographycomputed tomography (PET-CT), which showed metastatic lesions to the liver, abdominal lymph nodes, the inguinal regions, the retroperitoneum, the cervical lymph nodes, the left gluteus maximus muscle, and the vertebra prominence (C7). The progression-free survival (PFS) was eight mouths. The patient received four cycles of FOLFIRI plus bevacizumab from October to December 2019. A CT scan showed that the metastatic lymph nodes were smaller, but the skin nodules had increased in size and involved the skin of the hypogastrium, left thigh, bilateral groin, and perineum. These nodes mixed together, and formed tiny open sores, or ulcers, on the surface of the nodes (Figure 2B). A part of the rash was bleeding and painful. The PFS was $<1$ month. Skin biopsies were obtained in January 2020 (Figure 3A). The pathologic diagnosis of skin nodule was metastatic rectal adenocarcinoma. The epithelium was composed of columnar cells of pleomorphic vesicular nuclei with more than one nucleolus and frequent atypical mitotic figures. The stroma presented a mixed inflammatory reaction of lymphocytes, neutrophils, and eosinophils, fibrosis, and ecstatic vessels. Immunohistochemical analysis showed the following: CDX2(-) (Figure 3B); SATB2(+) (Figure 3C); CK7(-) (Figure 3D); CK20(+); TTF-1(-); Pax-8(-); MLH1(+); MSH2(+); MSH6(+); PMS2(+); GATA-3(-); Her-2(-); CD56(-); CgA (-); Syn (-); GCDFP-15(-); HMB45(-); and Ki67 $(+80 \%)$. The patient had received two cycles of FOLFIRI plus cetuximab and vemurafenib since January 2020. After treatment with FOLFIRI plus cetuximab and vemurafenib, the cutaneous nodules decreased in size (Figure 2C). The patient had severe electrolyte disturbances and depression and opted for palliative care (Figure 4).

\section{Details of Pathologic Examination}

The specimens were collected and submitted to our dermatopathology laboratory, formalin-fixed, and paraffin-embedded. The diagnosis had been established by examination of hematoxylin and eosin-stained sections and supplemented with appropriate immunostains (CK7, CK20, GCDFP15, Her-2, HMB45, SATB2, MLH1, MSH2, MSH6, PMS2, CD56, CgA, Syn, and Ki67).

\section{Summary of Pathologic Findings}

A skin biopsy was obtained from a lesion and the histopathologic examination showed a malignant neoplasm composed of well-formed ductal structures that had infiltrated the dermis. The findings were compatible with 
A

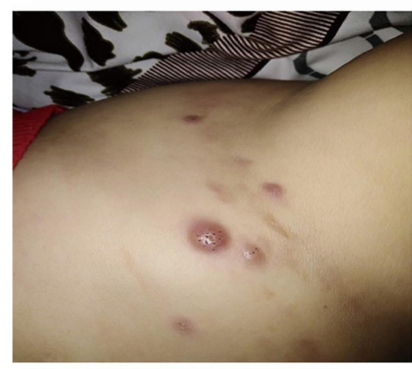

B

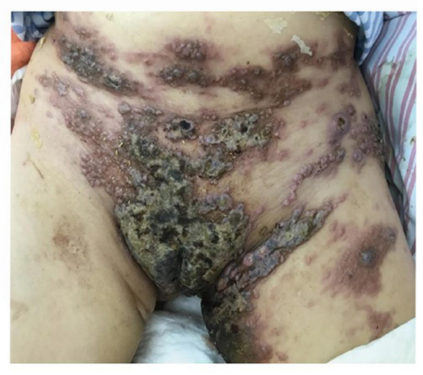

C

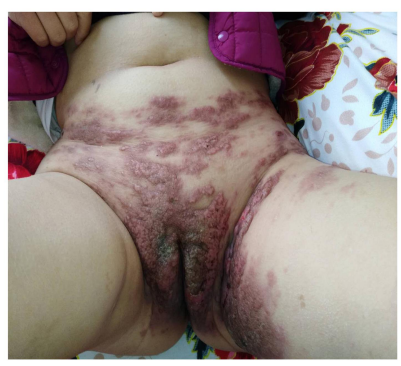

Figure 2 The evolution of skin metastases.

Notes: In September 2019, the skin of the hypogastrium and perineum was red and swollen with a rubbery appearance, a rash-like swelling on the surface, and local fusion (A); In December 2019, the skin nodules had increased in size and involved the skin of the hypogastrium, left thigh, bilateral groin, and perineum. These nodes mixed together, and formed tiny open sores, or ulcers, on the surface of the nodes (B); After palliative treatment with FOLFIRI plus cetuximab and vemurafenib, the cutaneous nodules decreased in size $(\mathbf{C})$.

A

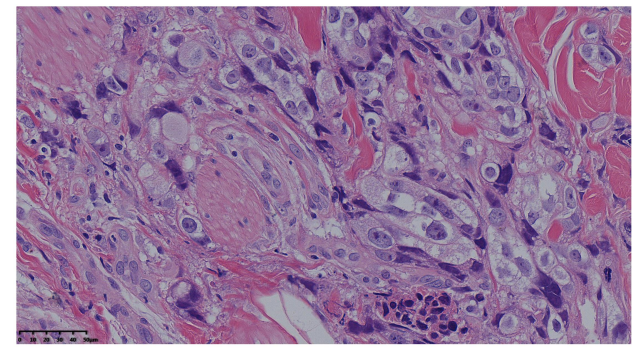

C

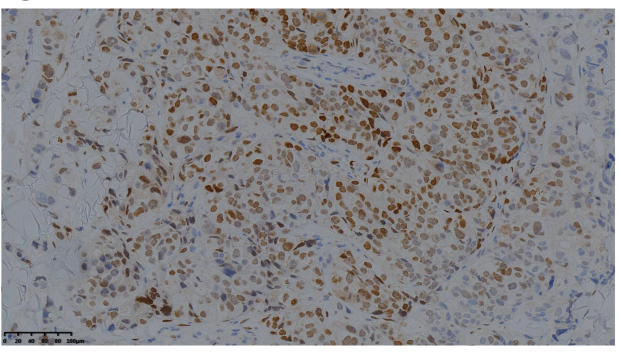

B

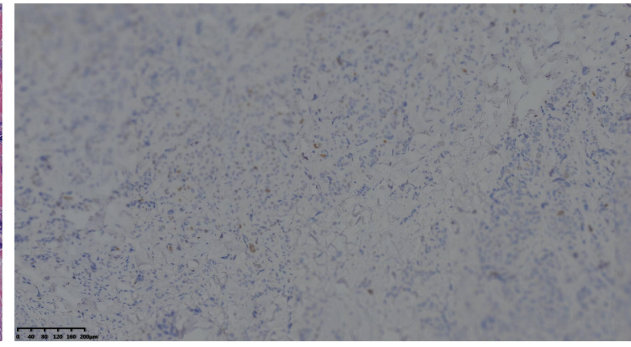

D

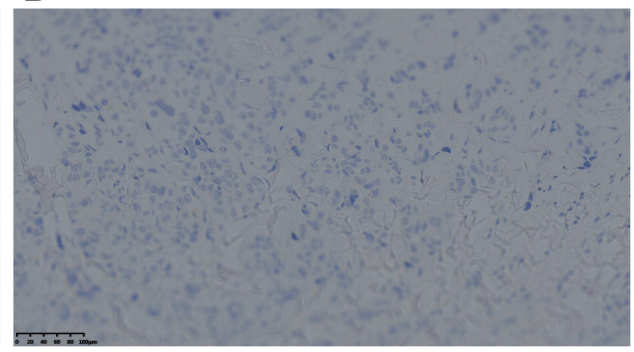

Figure 3 Pathological findings of rectal carcinoma skin metastases.

Notes: The pathologic diagnosis of skin nodule was metastatic rectal adenocarcinoma (hematoxylin and eosin staining, $\times 40)(\mathbf{A})$; Negative CDX2 staining in presence of skin metastases of rectal adenocarcinoma (DAB, $\times 10)(B)$; Positive SATB2 staining in presence of skin metastases of rectal adenocarcinoma (DAB, $\times 20)(C)$; Negative CK7 staining in presence of skin metastases of rectal adenocarcinoma (DAB, $\times 20)(D)$.

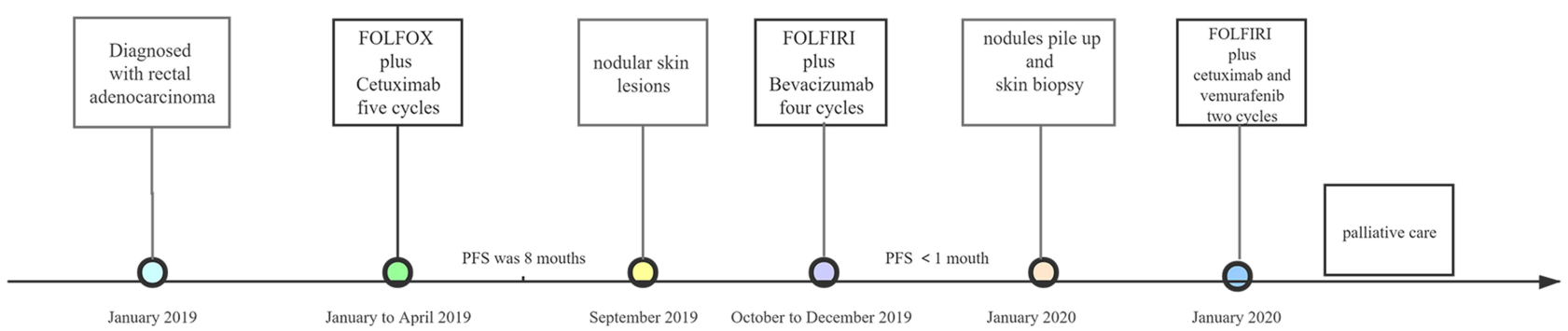

Figure 4 Timeline of diagnosis and treatment of the patient. 
metastatic adenocarcinoma (Figure 3A). The immunohistochemical examination was positive for SATB2 (Figure 3C), and negative for CDX2 (Figure 3B) and cytokeratin 7 (CK7) (Figure 3D).

\section{Discussion}

The most common site of metastasis of rectal cancer is the liver, followed by the lungs and bone. Metastasis to sites other than the liver and lungs are uncommon, and if they occur it is usually in the setting of extensive liver and lung metastases. Although colorectal cancer usually metastasizes to the liver and lungs, the skin may be involved in approximately $5 \%$ of the cases, and they constitute the 7 th most common cancer in women and the 3rd in men with skin involvement. ${ }^{3}$ As with other visceral organs, skin involvement is considered to be a sign of advanced stage disease in colorectal cancer. ${ }^{2}$ Through review and analysis of rectal cancer cases with cutaneous metastases, $\mathrm{Deha}^{7}$ reported a mean age of 55.5 years. Nineteen of the patients were male, the histology was based on adenocarcinoma, and 54\% of the patients had high-risk features, such as mucinous $(n=7)$, signet ring cell $(n=4)$, and poorly differentiated $(n=4)$. Most of the patients were stage III $(n=10)$ and IV $(n=11)$. In terms of the distribution of cutaneous metastases, perineal skin metastases were more common $(n=11)$. Some patients had multiple skin metastases, but the face and limbs were rarely affected. ${ }^{7}$ Cutaneous metastases usually present as nodules, ulcers, cellulitis-like lesions, and fibrosis. In this case, the cutaneous metastases had a rubbery appearance with a rash-like swelling and subcutaneous small nodules. On average, cutaneous metastases occur 4.9 years after colorectal cancer diagnosis, but a 15-year interval was noted in one case. ${ }^{2}$ Cutaneous metastases may also be present at the time of diagnosis, but this is extremely rare. Riyadh Hakami ${ }^{8}$ report a patient diagnosed with rectal cancer associated with extensive skin metastasis to the perineum and inguinal area, who carried the mutation c.34G>T (p. G12C) in exon 2 of the K-RAS oncogene. Besides, Amarjothi $\mathrm{JMV}^{9}$ report a 25 -year-old man with rectal adenocarcinoma with multiple peritoneal metastases, and it is not clear whether there was a BRAF mutation. However, as in our case, cutaneous metastases may also occur dramatically over a relatively short period of time. In our patient, the cutaneous metastases occurred after 8 months of treatment. Several mechanisms may explain their occurrence, such as direct tumor extension, dissemination by the lymphatic and/ or hematogenous routes, invasion of the ligaments of embryologic origin, or implantation of exfoliated tumor cells at surgical resection. ${ }^{10} \mathrm{~A}$ lot of tumor emboli were found in the vessels of specimens of the primary site and cutaneous nodules in the case. This finding may support the mechanism of dissemination by the lymphatic and/or hematogenous routes. Cutaneous metastases signal a poor prognosis. Survival ranges from 3-18 months. ${ }^{2}$ Gazoni et al ${ }^{11}$ reported a series of 6 patients with concurrent diagnoses of advanced stage IV rectal cancer and skin metastases. None of the patients survived $>7$ months after diagnosis, regardless of the treatment modality. ${ }^{11}$ In view of the extensive lesions in our patient, which were not amenable to surgery, she received third-line chemotherapy. After treatment with FOLFIRI plus cetuximab and vemurafenib, the cutaneous nodules decreased in size. The patient had severe electrolyte disturbances and depression and opted for palliative care. She died in February 2020. Her overall survival was $<5$ mouths from the onset of skin nodules.

Activating mutations of BRAF are found in 5\%-9\% of colorectal carcinomas, with most occurring in a hotspot of amino acid position 600 by a missense substitution of valine by glutamic acid, which is known as the BRAF V600E mutation. ${ }^{5}$ Patients with BRAF-mutated MSS colorectal carcinomas are most often stage IV at presentation compared to patients with BRAF-mutated MSI-H and BRAF wild-type MSS colorectal carcinomas (32\% vs $8 \%$ vs $15 \%$, positive for $\mathrm{CDX} 2 \mathrm{P}<0.001) .{ }^{12} \mathrm{CK} 20$ and $\mathrm{CDX}-2$ are two of the most sensitive and specific markers of intestinal differentiation and are extremely useful immunohistochemical markers in correctly identifying adenocarcinomas of colorectal origin in the metastatic setting. BRAF-mutated MSS colorectal carcinoma often displays reduced CDX-2 and CDX-2 loss has been shown to be associated with a worse prognosis in patients with colorectal carcinoma. ${ }^{6}$ It is generally known that the presence of a BRAF V600E mutation is an unfavorable prognostic factor in patients with colorectal carcinoma. Christensen identified a potential association between BRAF V600E mutation and increased risk of cutaneous metastases. ${ }^{13}$ Loss of CDX2 expression was more common in BRAF mutated metastatic colorectal cancer. CDX2 loss was associated with BRAF mutation, and poor differentiation. ${ }^{14}$ On the other hand, Baba et al analyzed 621 colorectal cancers, CDX2 loss was detected in $29 \%$ tumors and associated with female gender, but not with BRAF. ${ }^{15}$ The weakness was that this case did not get genetically tested right away. More research is needed to explore the inner relationship hidden in the BRAF mutation, CDX2 loss, and skin metastases. In the future, a comprehensive molecular analysis of the primary and metastatic tissues from these patients is 
warranted to elucidate the molecular characteristics of poor prognosis and potential prognostic markers.

Among patients with colorectal cancer, skin involvement can be detected during the clinical course. Skin involvement can be seen at the time of diagnosis or during the course of treatment and is a sign of advanced stage. Therefore, in patients with newly diagnosed or undergoing treatment for cancer, newly formed nodules in or under the skin should raise suspicion of metastatic disease and a careful physical examination and biopsy should be performed if indicated. In addition, skin biopsy is required to early diagnosis of unhealed skin wounds, swelling, and other skin changes. Treatment can be arranged accordingly. We have shown that a BRAFmutated/MSS rectal carcinoma may have an aberrant CK20/ CDX2 immunophenotype. BRAF-mutated/MSS colorectal carcinoma is an important subtype for clinicians to be aware of as the immunophenotype is often different from the classic colorectal pattern and often presents with metastatic disease.

\section{Ethics Statement}

The written informed consent was obtained from the patient and the patient agreed to publish his details of his case and any accompanying images. This study was approved by the ethics committee of Affiliated Jinhua Hospital, Zhejiang University School of Medicine (Zhejiang, China).

\section{Disclosure}

The authors declare that they have no competing interests.

\section{References}

1. Riihimäki M, Hemminki A, Sundquist J, et al. Patterns of metastasis in colon and rectal cancer. Sci Rep. 2016;6:29765. doi:10.1038/ srep29765

2. Lookingbill DP, Spangler N, Sexton FM. Skin involvement as the presenting sign of internal carcinoma. A retrospective study of 7316 cancer patients. J Am Acad Dermatol. 1990;22:19-26. doi:10.1016/ 0190-9622(90)70002-Y
3. Reingold IM. Cutaneous metastases from internal carcinoma. Cancer. 1966;19:162-168. doi:10.1002/1097-0142(196602)19:2<162::aidcncr2820190204>3.0.co;2-a

4. Lookingbill DP, Spangler N, Helm KF. Cutaneous metastases in patients with metastatic carcinoma: a retrospective study of 4020 patients. J Am Acad Dermatol. 1993;29:228-236. doi:10.1016/01909622(93)70173-Q

5. Van Cutsem E, Köhne CH, Láng I, et al. Cetuximab plus irinotecan, fluorouracil, and leucovorin as first-line treatment for metastatic colorectal cancer: updated analysis of overall survival according to tumor KRAS and BRAF mutation status. $J$ Clin Oncol. 2011;29:2011-2019. doi:10.1200/JCO.2010.33.5091

6. Slik K, Turkki R, Carpén O, et al. CDX2 loss with microsatellite stable phenotype predicts poor clinical outcome in stage II colorectal carcinoma. Am J Surg Pathol. 2019;43:1473-1482. doi:10.1097/ PAS.0000000000001356

7. Dehal A, Patel S, Kim S, et al.: Cutaneous metastasis of rectal cancer: a case report and literature review. Perm J 20:74-78, 2016

8. Hakami R, Alali MN, Alshammari T, et al. A cutaneous metastasis of unresectable rectal adenocarcinoma: a case report and literature review. Int J Surg Case Rep. 2020;71:95-101. doi:10.1016/j. ijscr.2020.04.102

9. Amarjothi JMV, Villalan R, Jeyasudhahar J, et al. Interesting case of skin metastasis in colorectal cancer and review of literature. Case Rep Surg. 2018;2018.

10. Kauffman CL, Sina B. Metastatic inflammatory carcinoma of the rectum: tumor spread by three routes. Am J Dermatopathol. 1997;19:528-532. doi:10.1097/00000372-199710000-00107

11. Gazoni LM, Hedrick TL, Smith PW, et al. Cutaneous metastases in patients with rectal cancer: a report of six cases. Am Surg. 2008;74:138-140. doi:10.1177/000313480807400210

12. Landau MS, Kuan SF, Chiosea S, et al. BRAF-mutated microsatellite stable colorectal carcinoma: an aggressive adenocarcinoma with reduced CDX2 and increased cytokeratin 7 immunohistochemical expression. Hum Pathol. 2014;45:1704-1712. doi:10.1016/j. humpath.2014.04.008

13. Christensen TD, Palshof JA, Larsen FO, et al. Associations between primary tumor RAS, BRAF and PIK3CA mutation status and metastatic site in patients with chemo-resistant metastatic colorectal cancer. Acta Oncol. 2018;57:1057-1062. doi:10.1080/ 0284186X.2018.1433322

14. Aasebø K, Dragomir A, Sundström M, et al. CDX2: a prognostic marker in metastatic colorectal cancer defining a better BRAF mutated and a worse KRAS mutated subgroup. Front Oncol. 2020;10:8. doi:10.3389/fonc.2020.00008

15. Baba Y, Nosho K, Shima K, et al. Relationship of CDX2 loss with molecular features and prognosis in colorectal cancer. Clin Cancer Res. 2009;15:4665-4673. doi:10.1158/1078-0432.CCR-09-0401
OncoTargets and Therapy

\section{Publish your work in this journal}

OncoTargets and Therapy is an international, peer-reviewed, open access journal focusing on the pathological basis of all cancers, potential targets for therapy and treatment protocols employed to improve the management of cancer patients. The journal also focuses on the impact of management programs and new therapeutic agents and protocols on patient perspectives such as quality of life, adherence and satisfaction. The manuscript management system is completely online and includes a very quick and fair peer-review system, which is all easy to use. Visit http://www.dovepress.com/ testimonials.php to read real quotes from published authors. 